\title{
High Energy Neutrino Astronomy
}

\author{
V. Berezinsky
}

INFN - Laboratori Nazionali del Gran Sasso, I-67010 Assergi (AQ), Italy

\begin{abstract}
The short review of theoretical aspects of ultra high energy (UHE) neutrinos. The accelerator sources, such as Supernovae remnants, Gamma Ray Bursts, AGN etc are discussed. The top-down sources include Topological Defects (TDs), Superheavy Dark Matter (SHDM) and Mirror Matter. The diffuse fluxes are considered accordingly as that of cosmogenic and top-down neutrinos. Much attention is given to the cascade upper limit to the diffuse neutrino fluxes in the light of Fermi-LAT data on diffuse high energy gamma radiation. This is most general and rigorous upper limit, valid for both cosmogenic and top-down models. At present upper limits from many detectors are close to the cascade upper limit, and 5 yr IceCube upper limit will be well below it.
\end{abstract}

Keywords: High Energy Neutrinos, Diffuse Gamma Radiation, Ultra High Energy Cosmic Rays

\section{Introduction}

Many neutrino telescopes, using different technique of observation, were searching for High Energy (HE) cosmic neutrinos during last 30-40 years. Among existing neutrino telescopes there are deep underwater/ice detectors (Baikal, ANTARES, IceCube, NESTOR), Extensive Air Shower detectors (Auger and HiRes) and radio-telescopes: ANITA-lite, RICE, GLUE, FORTE and others. Even bigger projects include JEM-EUSO and Super-EUSO.

Why is there such great interest to HE neutrinos?

HE neutrinos can provide us with most important information in physics and astrophysics.

Detection of HE neutrinos from SN remnants will prove that these objects are sources of galactic cosmic rays $(\mathrm{CR})$ and the Standard Model of Galactic Cosmic Ray origin will be confirmed.

Jet models of Gamma Ray Bursts (GRBs) and Active Galactic Nuclei (AGN) can be proved.

Detection of cosmogenic neutrinos can clarify the origin of Ultra High Energy Cosmic Rays (UHECR) and determine the model of transition from galactic to extragalactic CRs.
Detection of neutrino jets with energies above $10^{20}$ $10^{21} \mathrm{eV}$ means discovery of Topological Defects, important objects in standard cosmology.

Registration of HE neutrinos from the center of the Sun or Earth indicate the annihilation of Dark Matter (DM) particles there.

Mirror matter can be discovered with help of oscillation mirror neutrinos into visible ones.

\section{Basics of HE neutrino astrophysics}

We summarize here some basic features of HE neutrino astrophysics.

\section{Production of UHE cosmic neutrinos}

occurs in $p p$ and $p \gamma$ collisions of UHE protons with the target nuclei and with low-energy photons. They can be also produced by annihilation of DM particles and by decays of superheavy particles. In all these cases neutrinos are produced in the chain of pion decays.

\section{UHE neutrino sources}

are subdivided into accelerator and top-down sources, where neutrinos are produced in decays and annihilation of heavy particles. The examples of such sources are 
given by annihilation of neutralinos in the Sun and Earth [1], by topological defects (for a review see [2]), which produce superheavy unstable particles, and by decays of quasi-stable superheavy DM particles [3].

Usually HE neutrinos are accompanied by other radiations, most notably by HE gamma-rays and cosmic rays (CR). There are, however, so called "hidden sources" where all accompanying radiations are strongly or fully absorbed. The examples of such objects are the Sun and Earth, in center of which neutralinos annihilate. Another ideal example is given by mirror matter, where all mirror particles interact with visible matter gravitationally, and only mirror neutrinos can oscillate into visible ones. The almost "hidden" source is given by the Stecker model [4] of AGN, where UHE photons and protons are mostly absorbed or confined, and only $\mathrm{HE}$ neutrinos emerge from there.

In [5] a hidden neutrino source is produced in evolution of stellar cluster at its contraction. Collisions of neutron stars at the center of a cluster produce a rarefied cavity filled by ultra-relativistic fireballs from colliding neutron stars. The cavity is surrounded by thick gas envelope produced by destructed stars. All HE particles, such as protons, gamma and X-rays are absorbed in the thick envelope, and only UHE neutrinos escape.

Recently, the ultra-relativistic jet surrounded by the envelope of collapsing star is considered [6] as hidden UHE neutrino source.

There are also some other more conventional examples of astrophysical hidden sources [7, 8].

\section{Neutrino detection}

includes four remarkable reactions:

Muon production $v_{\mu}+N \rightarrow \mu+$ all gives an excellent tool to search for the discrete sources, since directions of UHE muon and neutrino coincide.

Resonant production of W-boson, $\bar{v}_{e}+e \rightarrow W^{-} \rightarrow \bar{v}_{\mu}+\mu$ (the Glashow resonance [9]) and $\bar{v}_{e}+e \rightarrow W^{-} \rightarrow$ hadrons [10] have the large cross-sections. For the practical applications the latter reaction is more important since it results in production of monoenergetic showers with energy $E_{0}=m_{W}^{2} / 2 m_{e}=6.3 \times 10^{6} \mathrm{GeV}[10]$ and can be observable in IceCube and future $1 \mathrm{~km}^{3}$ underwater detectors.

Tau production in a detector, $v_{\tau}+N \rightarrow \tau+$ hadrons, is characterized by time sequence of three signals [11]: a shower from prompt hadrons, the Cherenkov light from $\tau$ and hadron shower from $\tau$-decay. UHE $v_{\tau}$ are absorbed less in the Earth due to regeneration: absorbed $v_{\tau}$ is converted into $\tau$, which decays producing $v_{\tau}$ again. Another remarkable phenomenon produced by UHE $\tau$ - neutrinos is Earth-skimming effect [12], due to which the Auger observatory obtained the upper limit on UHE $\tau$ - neutrino flux [13].

Z-bursts provide a signal from the space, caused by the resonant $Z^{0}$ production by UHE neutrino on DM neutrino [14], $v+\bar{v}_{\mathrm{DM}} \rightarrow Z^{0} \rightarrow$ hadrons. The energy of the detected neutrino must be tremendous: $E_{0}=$ $m_{Z}^{2} / 2 m_{v} \sim 10^{24} \mathrm{eV}$.

Neutrino oscillations

play the essential role. The neutrino flavors $\bar{v}_{e}$ and $v_{\tau}$ are inefficiently produced in the accelerator sources. The flavor oscillation $\bar{v}_{\mu} \leftrightarrow \bar{v}_{e}$ and $v_{\mu} \leftrightarrow v_{\tau}$ can equalize the fluxes of these neutrinos. The oscillation length $L(E)$ is given by

$$
L(E)=\frac{4 \pi E}{\Delta m^{2}}=8.0\left(\frac{E}{10^{10} \mathrm{GeV}}\right)\left(\frac{10^{-4} \mathrm{eV}^{2}}{\Delta m^{2}}\right) \mathrm{pc}
$$

where $\Delta m^{2}$ are $2.4 \times 10^{-3} \mathrm{eV}^{2}$ and $7.7 \times 10^{-5} \mathrm{eV}^{2}$ for atmospheric and solar neutrino oscillations, respectively. Thus oscillation length is very short for cosmic distances and neutrino oscillations are very efficient. If neutrino flux is produced by decays of pions and muons with ratio $\pi^{+} / \pi^{-}=1$, the initial neutrino flavor ratio is $v_{e}: v_{\mu}: v_{\tau}=1: 2: 0$, and the observed flavor ratio (after oscillation) is $v_{e}: v_{\mu}: v_{\tau}=1: 1: 1$ (equipartition). This is the case of neutrinos produced in $p p$-collisions. In case of $p \gamma$ pion production $\bar{v}_{e}$ can be strongly suppressed, but flavor equipartition after oscillation is approximately holds. Many cases of flavor ratios with different conditions (e.g. neutron decay and survival, muon survival etc are studied in [15].

The matter neutrino oscillations in the sources and in the Earth can also occur (see e.g. [6]).

HE neutrinos from early universe.

One might think (and many did think) that large neutrino fluxes can be produced at cosmological epochs with large red shift $z$, e.g. due to decay of superheavy particles and production by topological defects. In fact, this possibility is disfavored [16, 17] by absorption of HE neutrinos and by nucleosynthesis bound on their fluxes. Neutrinos are absorbed in $v \bar{v}$ collisions with bigbang neutrinos and horizon of observation for neutrinos with energy $E_{\nu 0}$ (at present) is given by redshift

$$
z_{\mathrm{abs}}=7.9 \times 10^{4}\left(E_{\nu 0} / 1 \mathrm{TeV}\right)^{-1 / 3} \text {. }
$$

Neutrino fluxes produced at large z (e.g. by topological defects) are strongly restricted by production of $D$ and ${ }^{3} \mathrm{He}$ at the epochs after Big Bang nucleosynthesis. Neutrinos cause e-m cascades and $\mathrm{MeV}$ photons from these cascades produce $\mathrm{D}$ and ${ }^{3} \mathrm{He}$ in collisions with ${ }^{4} \mathrm{He}$ nuclei. 


\section{Astrophysical (accelerator) sources}

Protons are assumed to be accelerated mostly by the shocks and produce neutrinos in $p p$ and $p \gamma$ collisions. HE neutrinos from SN remnants

Detection of HE neutrinos from SN remnants is one of the most important tasks of HE neutrino astronomy, and this task looks perfectly realistic for IceCube and other $1 \mathrm{~km}^{3}$ detectors. Acceleration of protons and nuclei in SN remnants (SNRs) is the basic element of the Standard Model for Galactic CR (see e.g. [18]). These sources successfully explain the spectra and fluxes of CR observed in our Galaxy, and the knee is interpreted as the end of Galactic CR. Accelerated protons interacting with the gas in a SNR must emit gamma-rays and neutrinos through production and decays of neutral and charged pions. HE gamma rays are detected from several SNRs, but from most of them the signal is compatible with the bremsstrahlung or Inverse Compton production by HE electron. There are in some cases the indications to the hadronic gamma-rays (i.e. produced by neutral pions born in $p p$-collisions), however in all these cases one still may argue in HE electron production of the observed signal. Detection of HE neutrino signal gives unambiguous prove of pion production by accelerated protons.

There are at least two SN remnants, W28 and W44, for which the evidence for hadronic gamma-ray signal is rather strong. For both of them gamma-radiation is produced in a nearby dense molecular cloud. Gamma radiation from W28 was observed by AGILE detector at $E>400 \mathrm{MeV}$ [19], and by Fermi-LAT [21] and H.E.S.S [20] at higher energies. The proof is based on spectral characteristics and molecular cloud as the target. The case of W44 observed by Fermi-LAT is similar: gamma-radiation is observed from dense cloud ring around W44 [22].

SNRs discussed above are candidates for detectable neutrino fluxes by IceCube. Another candidate for the detected hadronic gamma-rays SNR RX J1713.7-3946 was analyzed in [23] for detectable neutrino flux. The authors accurately recalculated the observed gammaray flux, assuming its hadronic origin, to HE neutrino flux, and found that it corresponds to 2 - 3 events per year at energy above $1 \mathrm{TeV}$ for a detector as IceCube. Gamma Ray Bursts.

GRBs are most exiting sources of UHE neutrinos. There are two mechanisms of HE neutrino generation. In the first one [24] particles are accelerated by external shock and neutrinos are produced in $p \gamma$ collisions with GRB photons behind the shock. In the second mechanism [25] protons are accelerated by internal shocks, with the spectrum assumed to be $\propto 1 / E^{2}$. Neutrinos are produced in $p \gamma$ collisions with GRB photons. All estimates are very transparent and follow from assumption that the energy outputs in GRB photons, accelerated protons and produced neutrinos are about the same: $W_{v} \sim W_{p} \sim W_{\mathrm{GRB}}$. Then the total number of neutrinos with energy $E$ per burst is

$$
N_{\nu}(E) \sim \frac{W_{\mathrm{GRB}}}{\ln E_{\max } / E_{\min }} E^{-2},
$$

where $E_{\min }$ and $E_{\max }$ are minimum and maximum acceleration energy, respectively. Now one can express the flux of neutrinos from a single GRB, in terms of neutrino fluence $S_{v}$ :

$$
F_{v_{\mu}+\bar{v}_{\mu}}(E)=\frac{1}{3} \frac{S_{v}}{\ln \left(E_{\max } / E_{\min }\right)} E^{-2},
$$

and calculate the number of muons produced in $1 \mathrm{~km}^{3}$ detector per burst as

$$
P_{\mu}=N_{n} \int d E F_{v_{\mu}+\bar{v}_{\mu}}(E) \sigma_{v N}(E)
$$

where $N_{n}=6 \times 10^{38}$ is the number of nucleons in the detector and $\sigma_{v N}$ is $v_{\mu} N$ - cross-section. For $S_{v} \sim$ $10^{-5} \mathrm{erg} / \mathrm{cm}^{2}$, with frequency of bursts as in Fermi GRB Monitor $\dot{N}_{b} \sim 500 \mathrm{yr}^{-1}$, one finds the number of produced muons with $E \geq 10 \mathrm{TeV}$ for $5 \mathrm{yr}$ as $P_{\mu} \dot{N}_{b} t \sim 0.06$, i.e. too low for detection by IceCube.

The diffuse flux is estimated in identical way through the local neutrino emissivity $\mathcal{L}_{v}(0)$ and evolutionary factor $k_{\mathrm{evol}}$

$$
J_{v_{\mu}+\bar{v}_{\mu}}(E)=\frac{1}{3} \frac{c H_{0}^{-1}}{4 \pi} \frac{\mathcal{L}_{v}(0)}{E^{2} \ln E_{\max } / E_{\min }} k_{\mathrm{evol}},
$$

where evolutionary factor is given by

$$
k_{\mathrm{evol}}=\int_{0}^{z_{\max }} \frac{d z}{(1+z)^{2}} \frac{f_{\mathrm{evol}}(z)}{\sqrt{(1+z)^{3} \Omega_{m}+\Lambda}} .
$$

For the evolutionary function $f_{\text {evol }}(z)$ we take the case of strong star-formation evolution from [26], which results in $k_{\text {evol }}=7.0$ as a maximum value. For the emissivity we use

$$
\mathcal{L}_{\nu}(0) \leq \mathcal{L}_{\mathrm{GRB}}(0)=0.6 \times 10^{43} \mathrm{erg} / \mathrm{Mpc}^{3} \mathrm{yr},
$$

where the local GRB emissivity is taken from [27]. The most recent estimate $0.5 \times 10^{43} \mathrm{erg} / \mathrm{Mpc}^{3} \mathrm{yr}[28]$ agrees with the value above. For more detailed discussion see [29].

As a result $E^{2} J_{v_{\mu}+\bar{v}_{\mu}}(E)=1.15 \mathrm{eV} / \mathrm{cm}^{2}$ s sr, i.e. 25 times lower than sensitivity of IceCube shown in Fig 4 
GRB neutrinos are detectable by IceCube in case the hadronic energy output is an order of magnitude higher than one observed in photons, but it further strengthens the energetic deficit in GRB models. From observational point of view the signature of neutrino observation is very reliable due to time and direction correlation with gamma-radiation.

\section{HE neutrinos from AGN jets.}

The models of HE neutrino production in the AGN jets are very similar to that for GRBs. The protons are accelerated by the multiple shocks in the AGN jets, especially in their inner parts. Neutrinos are produced in the collisions with photons from the accretion disc and from photons produced in the jet by accelerated electrons and protons. Neutrino flux from individual AGN is very small, but the diffuse flux is predicted to be detectable by IceCube detector. The estimates can be performed similar to those in subsection above. One can found the detailed calculations in [30, 31, 32].

\section{HE neutrinos from galaxy clusters [33].}

The clusters of galaxies are able to confine the UHE particles for a time exceeding the age of the universe. This is the key phenomenon which makes galaxy clusters the powerful sources of UHE neutrinos. The particles are accelerated in clusters by various mechanisms: in the normal galaxies by SN shocks, in AGN and cDgalaxies, in the process of galactic merging etc. The diffuse HE neutrino flux is determined entirely by basic parameters characterizing the clusters. In particular, for the lower limit of the diffuse flux provided by normal galaxies in a cluster with CR luminosity $L_{p}$ and generation index $\gamma_{g}$, both taken as ones in our galaxy, the diffuse flux is given as

$$
J_{v}(E) \propto L_{p} E^{-\gamma_{g}} \frac{N_{g}}{R_{\mathrm{cl}}^{3}} \xi \Omega_{b} \rho_{\mathrm{cr}},
$$

where $R_{\mathrm{cl}} \sim 2 \mathrm{Mpc}$ is the virial radius of a cluster, $N_{g} \sim 100$ is richness of a cluster, $\rho_{\text {cr }}$ is critical cosmological density, and $\xi \Omega_{b}$ is cosmological baryonic density provided by clusters. The flux is marginally detectable by IceCube.

\section{Non-accelerator neutrino sources}

These sources include objects with annihilation of DM (the Sun, Earth, cores of the galaxies), objects with the decays of SHDM particles (galactic halos) and TDs. In the last two cases neutrinos are produced in the

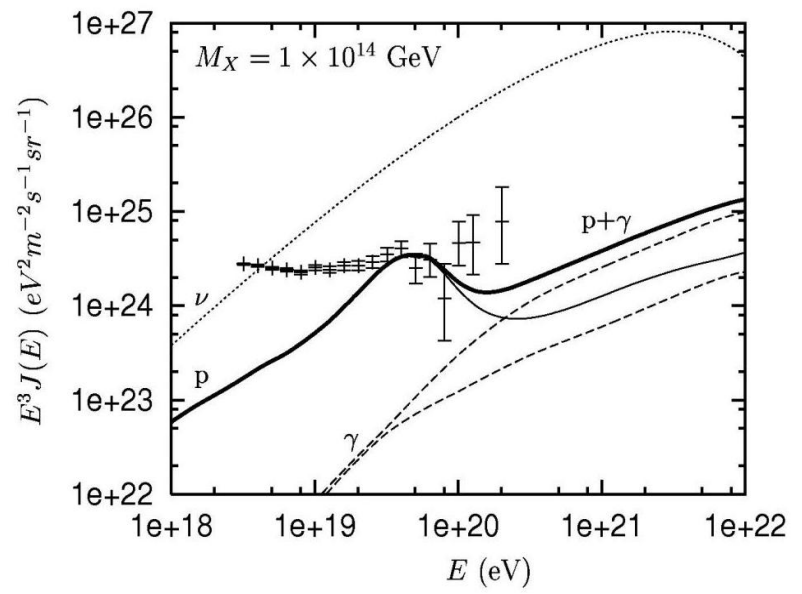

Figure 1: Diffuse all-flavor neutrino spectrum from necklaces for $m_{X}=1 \times 10^{14} \mathrm{GeV}[3]$. The thick curve gives $p+\gamma$ flux normalized to the AGASA UHECR data. If to normalize the proton flux in this figure by HiRes data all curves, including one labeled $v$ (neutrino flux), should be lowered by factor 3 - 5 .

decays of superheavy particles with the masses up to $M_{\mathrm{GUT}} \sim 10^{16} \mathrm{GeV}$. Neutrino spectrum can be approximately described at highest energies as $d E / E^{2}$.

\section{Neutralino annihilation in the Sun and Earth.}

Neutralino is the best motivated DM particle. Crossing the Sun or Earth a neutralino can loose its energy in collisions with nuclei and diminish its velocity below the escape velocity. If it happens, a neutralino becomes gravitationally trapped in the object, and loosing further their energies, neutralinos are accumulated in the center of a celestial body [1]. Annihilating there they produce short-lived hadrons, e.g. D-mesons, which decay to neutrinos. The process of annihilation strongly depends on neutralino mass and composition (mixture of basic fields: zino, bino and two higgsinos).

\section{Superheavy Dark Matter (SHDM)}

The first proposal of SHDM [34] was motivated by U1tra High Energy Cosmic Rays (UHECR) and by natural character of DM production at the epochs soon after inflation. In particular SHDM particles can be produced gravitationally [35], when the Hubble parameter $H(t)$ exceeds the particle mass $H(t) \gtrsim m_{X}$. The observed density of DM in the universe $\Omega_{\mathrm{cdm}} \approx 0.23$, determines the mass of the particle as $m_{X} \sim 10^{13} \mathrm{GeV}$. The SHDM particles (X-particles) can be stable (due to e.g. discrete gauge symmetry) or quasi-stable (due to superweak discrete gauge symmetry breaking). The energy spectrum of produced particles has approximately power-law form at the highest energies $\propto E^{-1.9}[3]$. The dominant decay particles are photons and neutrinos. As 


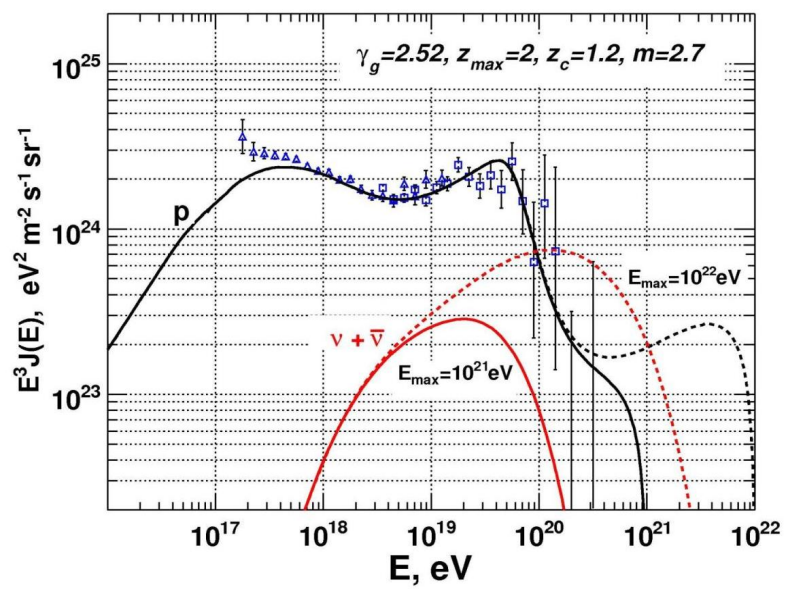

Figure 2: UHE neutrino flux in the dip model with AGN as the sources of UHECR. The cosmological evolution of AGN with $m=2.7$ up to $z_{c}=1.2$ is taken from X-ray observations of AGN. At larger $z$ the evolution is frozen up to $z_{\max }=2.0$. The fit of the dip is very good, though requires $\gamma_{g}=2.52$ different from the non-evolutionary case $m=0$. The neutrino fluxes are given for one neutrino flavor.

any cold DM, X-particles are accumulated in the halos of galaxies, in particular in our galaxy with overdensity $2.1 \times 10^{5}$. One can expect the detectable fluxes of UHE photons and neutrinos from the Galactic Center region.

Topological defects (TDs).

TDs are fundamental cosmological objects. They are produced in early universe due to symmetry breaking accompanied by the phase transitions. In many cases TDs become unstable and decompose to constituent fields, superheavy gauge and Higgs bosons (Xparticles), which then decay producing UHE neutrinos (see [2, 36] for the reviews).

Ordinary strings are produced by $U(1)$ symmetry breaking. There are several mechanisms by which ordinary strings can emit HE neutrinos: collapse of the string loops, self-intersection, annihilation of cusps, production and annihilation of tiny loops. In most cases produced neutrino fluxes are too low for detection. More promising scenario is given by the radiation of $\mathrm{X}$ particles through the cusp, a peculiar point where velocity reaches velocity of light. This point appears on a loop during each period. The points of the loop in the cusp region have distribution in values of the Lorentz factors $\Gamma$ from a maximum value in the cusp point to $\Gamma=1$. The particles escaping through a cusp region are boosted by these Lorentz factors. Recently, such case has been considered in [37]. The bosonic (Higgs) condensate in a string loop, emits Higgses through the cusp, and due to the Lorentz factor boost, these particles can reach the tremendous energies.

\section{Superconducting strings}

can be powerful sources of neutrinos. In a wide class of elementary particle models, strings behave like superconducting wires. The charge carriers are massless inside the string and superheavy outside. Moving through cosmic magnetic fields, such strings develop electric current. When the current reaches the critical value, the charge carriers escape from a string, turn into massive mode and decay. This process is strongly enhanced near the cusps due to the Lorentz boost. The decay products, in particular neutrinos, are emitted isotropically in a frame of cusp segment at rest, and propagate in the laboratory system as a very narrow jet with the opening angle $\vartheta \sim 1 / \Gamma$. This scenario was analyzed numerically in [38] with two main model features included. First, from all known structures of the universe, the excitation of electric current occurs most efficiently in clusters of galaxies for which the magnetic field reaches $B \sim 10^{-6} \mathrm{G}$ and filling factor $f \sim 10^{-3}$. Second, the symmetry breaking scale of order $10^{9}-10^{12} \mathrm{GeV}$ must be assumed for detectable neutrino fluxes. The typical Lorentz factor of the radiating cusp segment is calculated to be $\Gamma_{c} \sim 10^{12}$, and the maximum energy of emitted particle can reach $\Gamma_{c} \eta \sim 10^{22} \mathrm{GeV}$. The neutrino spectrum is assumed $\propto 1 / E^{2}$, similar to $\propto E^{-1.9}[3]$. The spectrum $E^{2} J_{v}(E)=$ const can be very close to the $E^{-2}$-cascade upper limit in Fig 4

\section{Necklaces (monopoles connected by string)}

are produced in the $G \rightarrow H \times U(1) \rightarrow H \times Z_{2}$ sequence of symmetry breaking, with each monopole being attached to two strings, and a loop reminds a necklace with monopoles playing the role of beads. In the process of evolution the strings shrink due to gravitational radiation and $M \bar{M}$ pairs in the necklace inevitably annihilate. This model is most plausible and well developed for UHE neutrino production. Diffuse neutrino flux from necklaces are shown in Fig. 1 according to calculations in [3].

\section{Cosmogenic neutrinos}

Starting from pioneering work [39] the fluxes of cosmogenic neutrinos have been calculated in many works [40] - [49]. The predicted fluxes differ very considerably, depending on the assumptions about mass composition of accelerated particles, on maximum energy of acceleration and on cosmological evolution of the sources. We present here the UHE neutrino fluxes calculated in the dip model for the observed UHECR [29. [50], assuming AGN as the sources [51]. This 


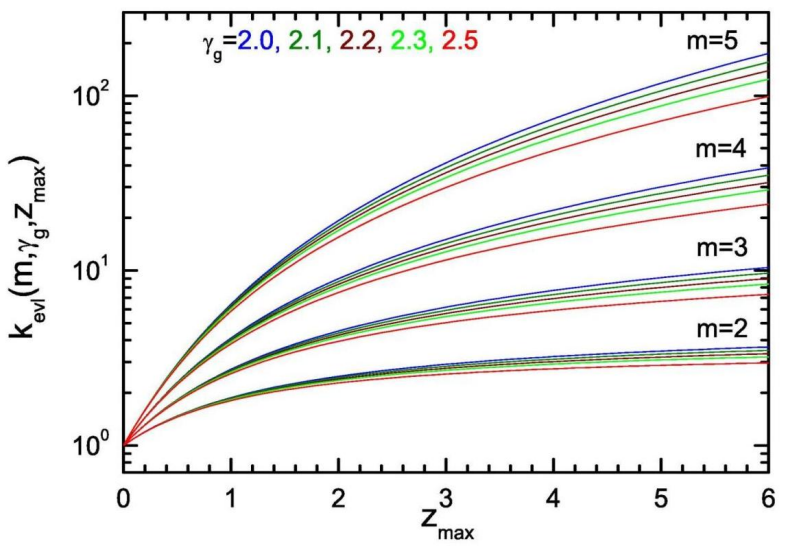

Figure 3: Evolution factor $k_{\mathrm{ev}}$, which describes increasing of neutrino flux due to cosmological evolution of the sources. The evolution factor is shown as function of $z_{\max }$ for different indices $\gamma_{g}$ and parameters of evolution $m$. The evolution factor $k_{\mathrm{ev}}$ is large for large $m$ and $z_{\max }$.

model is valid for the proton-dominated composition of UHECR, based on observations of HiRes. The pair production dip is a feature of interaction of extragalactic UHE protons propagating through CMB. It is caused by energy losses of protons due to $p+\gamma_{\mathrm{CMB}} \rightarrow e^{+}+e^{-}+p$ scattering. This feature in proton spectrum is well confirmed by observational data [29, 50].

To calculate neutrino flux produced by UHE protons it is enough to know the generation rate of UHE protons at each cosmological epoch. We take it as $Q(E)(1+z)^{m}$, where $Q(E) \propto E^{-\gamma_{g}}$ and $(1+z)^{m}$ describes the cosmological evolution of the sources up to some maximal redshift $z_{\max }$. In calculations we consider two cases: without evolution when we have only one free parameter, the generation index $\gamma_{g}$, and evolutionary scenario with three free parameters $\gamma_{g}, m$ and $z_{\max }$. We must fit the observed HiRes spectrum with one calculated at $z=0$. In non-evolutionary scenario the best fit with very good $\chi^{2}$ is given by $\gamma_{g}=2.7$. For the fit with evolutionary model we assume AGN as the sources and take the AGN evolution from X-ray observations [52]: $(1+z)^{m}$ with $m=2.7$ up to $z_{c}=1.2$, and frozen evolution from $z_{c}$ to $z_{\max }=2$. The generation index is fixed as $\gamma_{g}=2.52$ for the best fit of HiRes data (see Fig. 2). One may notice that the theoretical dip automatically describes the ankle at $E \approx 5 \times 10^{18} \mathrm{eV}$. The calculated one-flavor neutrino fluxes for the AGN evolutionary model are shown in Fig. 2 for two values of $E_{\max }$.

The evolution is able to increase strongly the neutrino flux. The increase of the flux is given by the evolution factor $k_{\mathrm{ev}}$, which depends on $m, z_{\max }$ and $\gamma_{g}$. This dependence is shown in Fig. 3

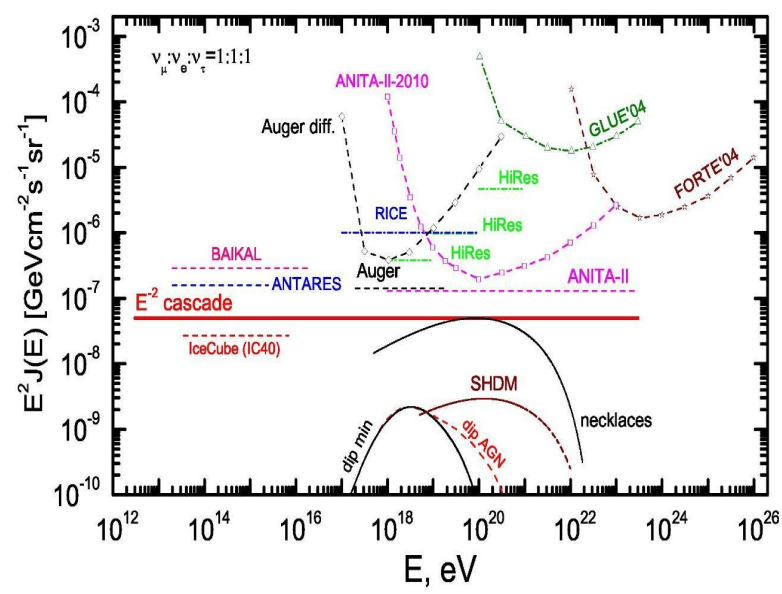

Figure 4: The experimental upper limits on UHE neutrino fluxes in comparison with e-m cascade upper limit in assumption of $E^{-2}$ generation spectrum (curve $E^{-2}$ cascade) and with predictions for cosmogenic neutrinos in the dip model (curves dip-min and dip-AGN), for neutrinos from necklaces and from SHDM. Neutrino fluxes from necklaces and SHDM are normalized by AGASA data, and for normalization by HiRes data the fluxes should be diminished by factor $3-5$. Neutrino flux from superconducting strings is given by $E^{2} J(E)=$ const and it can reach the upper limit ' $E^{-2}$ cascade'. Neutrino fluxes are given for three flavors.

\section{Cascade upper limit on diffuse neutrino flux}

The e-m cascade upper bound puts the rigorous upper limit on UHE neutrino flux [53, 8]. This limit, in contrast to WB upper limit [54], is valid for both accelerator and non-accelerator neutrinos. The production of neutrinos is accompanied by production of high energy photons and electrons from pion decays. Colliding with low-energy target photons, a primary photon or electron produces e-m cascade due to reactions $\gamma+\gamma_{\mathrm{tar}} \rightarrow e^{+}+e^{-}, e+\gamma_{\mathrm{tar}} \rightarrow e^{\prime}+\gamma^{\prime}$, etc. The cascade spectrum in its high-energy part is proportional to $E^{-2}$, which is very close to the EGRET observations in the range $10 \mathrm{MeV}-100 \mathrm{GeV}$ [55]. The observed energy density in this range is $\omega_{\text {EGRET }} \approx(2-3) \times 10^{-6} \mathrm{eV} / \mathrm{cm}^{3}$. The cascade energy density must be $\omega_{\text {cas }} \leq \omega_{\text {EGRET }}$, and it limits diffuse neutrino flux. The situation has dramatically changed with the new data of Fermi-LAT [56] on the flux and spectrum of diffuse extragalactic gamma-radiation. In comparison with EGRET this flux is lower and spectrum is steeper $\left(\propto E^{-2.4}\right)$. It results in stronger upper limit on the cascade energy density $\omega_{\text {cas }} \leq 5.8 \times 10^{-7} \mathrm{eV} / \mathrm{cm}^{3}$ [57], which severely diminishes the allowed UHE neutrino fluxes [57, 58]. The maximally allowed cascade energy density $\omega_{\text {cas }}^{\max } \approx$ $5.8 \times 10^{-7} \mathrm{eV} / \mathrm{cm}^{3}$ provides the upper limit on the integral UHE neutrino flux $J_{v}(>E)$ (sum of all flavors). It 
is given by chain of the following inequalities

$$
\omega_{\mathrm{cas}}>\frac{4 \pi}{c} \int_{E}^{\infty} E J_{v}(E) d E>\frac{4 \pi}{c} E \int_{E}^{\infty} J_{v}(E) d E
$$

where $\omega_{\text {cas }}<\omega_{\text {cas }}^{\max }$, and the integral in rhs of Eq. (6) gives the integral spectrum of neutrinos $J(>E)$. Thus, this equation gives the upper limit on the integral neutrino flux, which can be expressed in terms of the upper limit on differential neutrino spectrum $J_{v}(E)$ as

$$
E^{2} J_{v}(E)<\frac{c}{4 \pi} \omega_{\mathrm{cas}}^{\max } .
$$

Eq. (1) gives the rigorous upper limit on the neutrino flux. It is valid for neutrinos produced by HE protons, by topological defects, by annihilation and decays of superheavy particles, i.e. in all cases when neutrinos are produced through decay of pions and kaons. It holds for arbitrary neutrino spectrum falling down with energy. If one assumes some specific shape of neutrino spectrum, the cascade limit becomes stronger. For $E^{-2}$ generation spectrum, which is used for analysis of observational data one obtains the stronger upper limit. Given for three neutrino flavors it reads

$$
E^{2} J_{v}(E) \leq \frac{c}{4 \pi} \frac{\omega_{\mathrm{cas}}^{\max }}{\ln \left(E_{\max } / E_{\min }\right)},
$$

This upper limit is shown in Fig. 4

The most interesting energy range in Fig. 4 corresponds to $E_{v}>10^{21} \mathrm{eV}$, where acceleration cannot provide protons with sufficient energy for production of these neutrinos. At present the region of $E_{v}>10^{21} \mathrm{eV}$, and $E_{v} \gg 10^{21} \mathrm{eV}$ is considered as a signature of topdown models, which provide these energies quite naturally.

As one can see from Fig. 4 the observational upper limit for IceCube after $5 \mathrm{yr}$ of observations will be below the cascade upper limit. Crossing it, this detector will enter the physically allowed region of neutrino fluxes, and it can be regarded as historical event. The WB upper limit is not relevant for UHE neutrinos: it is not valid for top-down scenarios because proton production is strongly suppressed for top-down sources, and it is very uncertain for cosmogenic neutrinos, where for the same proton flux the fluxes of accompanying neutrinos may differ by one-two orders of magnitudes (see Fig. 2). However, the WB upper bound remains the convenient low-flux benchmark for detection of neutrino fluxes.

\section{Mirror matter and mirror neutrinos.}

Mirror neutrinos give the only example of fluxes not limited by the cascade upper limit.
The concept of mirror matter, as first was suggested by Lee and Yang [59], consists in existence of sector of matter fully symmetric with ordinary one and generated by space-reflection transformation. Kobzarev, Okun and Pomeranchuk [60] added the basic assumption that these two sectors communicate only gravitationally. The gravitational interaction results in mixing of mirror and ordinary neutrinos and their oscillations [61]. In two-inflaton cosmological model [62] the mirror matter is suppressed, while mirror TDs can strongly dominate. Mirror TDs copiously produce mirror neutrinos with extremely high energies, which oscillate into visible ones, while all other mirror particles, which accompany production of mirror neutrinos, remain invisible for our detectors. Therefore, the upper limits on HE neutrinos in our world do not exist and their fluxes can be above the upper limit shown in Fig. 4. Neutrinos from TDs typically have very high energies and one can see that fluxes of discussed neutrinos are now very severely constrained by ANITA-lite data [63].

\section{Conclusions}

UHE neutrinos are expected to solve many problems in astrophysics and cosmology. Detection of HE neutrinos from SNRs by IceCube is needed for confirmation of Standard Model for GCRs. UHE neutrinos from GRBs will clarify the nature of these most unusual and controversial objects. As far as $\mathrm{HE}$ radiation is concerned, this is true for AGN, too.

The diffuse UHE neutrino radiation is presented by cosmogenic and top-down neutrinos, in particular neutrinos from TDs. The fundamental problem of astrophysics involved in cosmogenic neutrinos is acceleration of particles. The shock acceleration at present knowledge of its theory cannot provide $E_{\max }$ higher than $10^{21}-10^{22} \mathrm{eV}$, and thus energies of cosmogenic neutrinos cannot exceed $3 \times 10^{20} \mathrm{eV}$. TDs naturally produce neutrinos emitted from cusps with energies by many orders of magnitude higher. Detection of neutrinos with these energies mean discovery of new physics.

Cascade upper limit is very general bound valid for both cosmogenic and top-down neutrinos. This upper limit became stronger with new Fermi-LAT data on extragalactic HE diffuse gamma-radiation. From all existing detectors only IceCube reached the sensitivity below the cascade upper limit (see Fig. 4) and entered the physically allowed region for detectable neutrino fluxes. It can be considered as historical event for HE neutrino astronomy.

The flux of cosmogenic neutrinos can be large only in case UHECR are proton-dominated. Even in this case 
the flux is detectable if maximum acceleration energy $E_{\max }$ is large and sources have strong cosmological evolution (see [57, 58]).

Cosmogenic neutrinos of highest energies are detectable by future experiment JEM-EUSO in rather extreme models with large $E_{\max }$ and strong cosmological evolution (see [57, 58]).

The search for UHE neutrinos in any case is a search for a new physics, either for astrophysics (the new acceleration mechanisms and cosmological evolution of the sources) or for topological defects, mirror topological defects and superheavy dark matter.

\section{Acknowledgments}

I am grateful to Askhat Gazizov for many discussions and help in preparing the figures.

\section{References}

[1] W.H. Press and D.N. Spergel, Ap.J 296 (1985) 679.

[2] P. Bhattacharjee and G. Sigl, Phys. Rep. 327 (2000) 109.

[3] R. Aloisio, V. Berezinsky, M. Kachelrieß, Phys. Rev. D 69 (2004) 094023.

[4] F.W. Stecker et al, Phys. Rev. Lett., 66 (1991) 2697;

[5] V. Berezinsky and V. Dokuchaev, Astrop. Phys. 15 (2001) 87.

[6] S. Razzaque, A.Yu. Smirnov, JHEP 03 (2010) 031.

[7] V. Berezinsky, C. Castagnoli, P. Galeotti, Niovo Cim. C, 8 (1985) 185.

[8] V. S. Berezinsky, S. V. Bulanov, V. A. Dogiel, V. L. Ginzburg and V. S. Ptuskin, Astrophysics of Cosmic Rays, North-Holland 1990.

[9] S.L. Glashow, Phys. Rev. D 118 (1960) 316.

[10] V.S. Berezinsky and A.Z. Gazizov, JETP Lett., 25 (1977) 254.

[11] J. Learned and S. Pakvasa, Astropart. Physics, 3 (1995) 267.

[12] D. Fargion, Ap.J. 570 (2002) 909.

[13] J. Abraham et al. [The Pierre Auger Collaboration], Phys. Rev. Lett. 100 (2008) 211101.

[14] T. Weiler, Astropart. Phys. 11 (1999) 303.

[15] S. Pakvasa, W. Rodejohann, T. Weiler, JHEP, 0802:005 (2008).

[16] V. Berezinsky, Nucl. Phys. B 380 (1992) 478

[17] R. Protheroe, T. Stanev, V. Berezinsky, Phys. Rev. D 51 (1995) 4134

[18] E.G. Berezhko and H.J. Völk, Ap.J. 661 (2007) L175.

[19] A. Giuliani et al [AGILE Collaboration], arXiv:1005.0784

[20] F. Aharonian et al, Astron.Astroph. 481 (2008) 401.

[21] A.A. Abdo et al [Fermi Collaboration] arXiv: 1005.4474.

[22] A.A. Abdo et al [Fermi Collaboration], Science, 327 (2010) 1103 .

[23] F. Vissani, G. Pagliaroli, F. Villante, arXiv:0912.4580

[24] M. Vietri, Phys. Rev. Lett. 80 (1998) 3690.

[25] E. Waxman and J. Bahcall, Phys. Rev. Lett. 78 (1997) 2292.

[26] S.D. Wick, C.D. Dermer, A.Atoyan, Astropart. Phys. 21 (2004) 125.

[27] Maarten Schmidt, Astroph. J. 559 (2001) L79 and private communication 2002.

[28] D. Eichler, D. Guetta, M. Pohl, arXiv:1007.3742

[29] V. Berezinsky, A. Gazizov, S. Grigorieva, Phys. Rev. D 74 (2006) 043005
[30] A. Atoyan and C.D. Dermer, Ap. J. 586 (2003) 79

[31] A. Atoyan and C.D. Dermer, New Astron. Rev. 48 (2004) 381.

[32] C.D. Dermer et al., New J.Phys. 11 (2009) 065016.

[33] V. Berezinsky, P. Blasi, V. Ptuskin, Astroph. J. 487 (1997) 529; P. Blasi and S. Colafrancesco, Astropart. Phys. 122 (1999) 169; D. DeMarco et al., Phys. Rev. D 73 (2006) 043004.

[34] V. Berezinsky, M. Kahelriess, and A. Vilenkin, Phys. Rev. Lett., 79 (1997) 4302;

V.A. Kuzmin and V.A. Rubakov, Yad. Fiz. 61 (1998) 1122; D.J.H. Chung, E.W. Kolb, and A. Riotto, Phys. Rev. D 60 (1999) 063504.

[35] D.J.H. Chung, E.W. Kolb, and A. Riotto, Phys. Rev. D 59 (1998) 023501;

V. Kuzmin and I. Tkachev, JETP Lett. 68 (1998) 271

[36] V. Berezinsky, Nucl. Phys. B (Proc. Suppl) 75A (1999) 119.

[37] T. Vachaspati, arXiv:0911.2655

[38] V. Berezinsky, K. Olum, E. Sabancilar and A. Vilenkin, Phys. Rev. D 80 (2009) 023014

[39] V. S. Berezinsky and G. T. Zatsepin, Phys. Lett., B 28 (1969) 423; V. S. Berezinsky and G. T. Zatsepin, Soviet Journal of Nuclear Physics 11 (1970) 111.

[40] R. Engel, D. Seckel and T. Stanev, Phys. Rev., D 64 (2001) 093010.

[41] O. E. Kalashev, V. A. Kuzmin, D. V. Semikoz and G. Sigl, Phys. Rev. D 66 (2002) 063004.

[42] Z. Fodor, S. Katz, A. Ringwald and H. Tu, JCAP 0311 (2003) 015 .

[43] V. Berezinsky, A. Gazizov and S. Grigorieva, Proc. of 11th Int. Workshop "Neutrino Telescopes”ed. M. Baldo-Ceoilin p. 339 (2005), astro-ph/0509675

[44] D. Hooper, A. Taylor, S. Sarkar, Astropart.Phys. 23 (2005) 11.

[45] M. Ave et al., Astropart.Phys. 23 (2005) 19.

[46] D. Seckel, T. Stanev, Phys. Rev. Lett. 95 (2005) 141101 (2005).

[47] T. Stanev, astro-ph/0808.1045.

[48] D. Allard et al., JCAP 0609 (2006) 005

[49] K. Kotera, D. Allard, A. Olinto, arXiv:1009.1382

[50] V. Berezinsky, A. Gazizov, S. Grigorieva, Phys. Lett. B 612 (2005) 147.

[51] V. Berezinsky, A. Gazizov, S. Grigorieva, arXiv:astro$\mathrm{ph} / 0210095$

[52] Y. Ueda et al, Astroph. J., 598 (2003) 886 A. J. Barger et al, Astron. J., 129 (2005) 578.

[53] V. Berezinsky and A. Smirnov, Ap. Sp. Sci., 32 (1975) 461.

[54] E. Waxman and J. Bahcall, Phys. Rev. D 59 (1999) 023002.

[55] P. Sreekumar et al. [EGRET collaboration], Astroph. J. 494 (1998) 523.

[56] A.A. Abdo et al., [Fermi-Lat collaboration], Phys. Rev. Lett. 104 (2010) 101101.

[57] V. Berezinsky, A. Gazizov, M. Kachelrieß, S. Ostapchenko, arXiv:1003.1496

[58] M. Ahlers et al., Astropart. Phys. 34 (2010) 106; arXiv: 1005.2620

[59] T.D. Lee and C.N. Yang, Phys. Rev. 104 (1956) 254

[60] V. Kobzarev, L. Okun, I. Pomeranchuk, Sov. J. Nucl. Phys. 3 (1966) 837.

[61] Z.G. Berezhiani and R.N. Mohapatra, Phys. Rev. D 52 (1995) 6607 ,

V. Berezinsky, M. Narayan, and F. Vissani, Nucl. Phys. B 658 (2003) 254.

[62] V. Berezinsky and A. Vilenkin, Phys. Rev. D 62 (2000) 083512.

[63] P. Gorham et al. [ANITA-lite collaboration] Phys. Rev. D 82 (2010) 022004 